\title{
Recurrent ulnar nerve entrapment at the elbow: Correlation of surgical findings and 3-Tesla magnetic resonance neurography
}

\author{
Avneesh Chhabra MD¹, Vibhor Wadhwa MBBS¹, Rashmi S Thakkar MD¹, John A Carrino MD MPH ${ }^{1}$, A Lee Dellon MD²
}

A Chhabra, V Wadhwa, RS Thakkar, JA Carrino, AL Dellon. Recurrent ulnar nerve entrapment at the elbow: Correlation of surgical findings and 3-Tesla magnetic resonance neurography. Can J Plast Surg 2013;21(3):186-189.

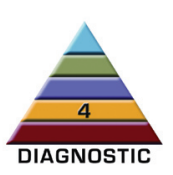

The authors describe the correlation between 3-Tesla magnetic resonance neurography (MRN) and surgical findings in two patients who underwent multiple previous failed ulnar nerve surgeries. MRN correctly localized the site of the abnormality. Prospectively observed MRN findings of perineural fibrosis, ulnar nerve re-entrapment abnormalities, medial antebrachial cutaneous neuroma and additional median nerve entrapment were confirmed surgically.

Key Words: 3T MRN; MR neurography; Subcutaneous transposition; Submuscular transposition; Ulnar nerve entrapment

Cubital tunnel syndrome is the second most common peripheral nerve compression syndrome of the upper extremity following carpal tunnel syndrome (1). Surgical release of the entrapped ulnar nerve is commonly treated using neurolysis with or without anterior subcutaneous or submuscular transposition (2). Persistent symptoms following repeated ulnar nerve release may occur due to failure of the initial surgery, re-entrapment, iatrogenic nerve injury or compression of the lower trunk of the brachial plexus, whereas focal pain at the surgical site can occur due to a neuroma of the medial antebrachial cutaneous nerve (3). High-field, 3-Tesla (3T) magnetic resonance neurography $(\mathrm{MRN})$ is a noninvasive technique that can be used to localize the site of a peripheral nerve abnormality by assessing the nerve's anatomy and continuity, as well as evaluating intraneural and perineural abnormality, local mass lesion and regional muscle denervation changes. MRN supplements information obtained from electrodiagnostic examinations and also provides information not attainable from the latter, such as localization of neural and perineural abnormalities, grading the degree of nerve entrapment or injury, and exclusion of other joint derangement abnormalities that could cause similar symptomatology. The diagnostic accuracy of MRN is excellent in larger nerves, such as the sciatic nerve, and good in smaller nerves such as the common peroneal nerve, with good to excellent interobserver reliability $(4,5)$. MRN findings in failed cubital tunnel release cases have not been reported. The authors report two patients in whom 3T MRN performed preoperatively predicted ulnar nerve reentrapment and other findings that were subsequently confirmed intraoperatively.

Patient 1

\section{CASE PRESENTATIONS}

A 46-year-old woman with a history of multiple (five) ulnar nerve surgeries at the right elbow (three neurolysis and one anterior subcutaneous followed by submuscular transposition) presented many months

\section{La compression récurrente du nerf cubital au coude : corrélation des observations chirurgicales et de la neurographie par résonance magnétique 3-Tesla}

Les auteurs décrivent la corrélation entre la neurographie par résonance magnétique (NRM) 3-Tesla et les observations chirurgicales chez deux patients qui avaient déjà subi plusieurs opérations non réussies du nerf cubital. La NRM a permis de déterminer le foyer de l'anomalie. Les observations prospectives des résultats de la NRM faisant foi d'une fibrose périneurale, d'anomalies de recompression du nerf cubital, d'un névrome cutané de la partie médiale de l'avant-bras et d'une compression supplémentaire du nerf médian ont été confirmées lors de l'opération. after the submuscular transposition with persistent lower posteromedial arm and forearm pain, tingling sensation in the ring and little fingers, and weakness in pinch and grip. The surgeries were performed by multiple surgeons in different surgical centres. The Tinel sign was positive at the level of the elbow joint, both proximal to the elbow and immediately distal to the elbow. There was decreased two-point discrimination in the ulnar nerve distribution. Electromyography (EMG) showed moderate ulnar neuropathy. 3T MRN (Siemens, Germany) of the brachial plexus, upper arm and elbow was ordered without contrast to exclude a structural lesion along the course of the nerve or 'thoracic outlet syndrome' and to evaluate the status of the ulnar nerve at the elbow. Both two-dimensional and three-dimensional pulse sequences were used.

The MRN showed normal appearance of the brachial plexus (not shown). The ulnar nerve in the proximal and mid arm was normal. Distally, as the ulnar nerve exited the medial intermuscular septum, there was mild perineural fibrosis with mild hyperintensity and enlargement of the ulnar nerve (Figures 1A to 1C), suggestive of entrapment. There were no enlarged fascicles or focal neuroma. The ulnar nerve was mildly flattened as it traversed beneath the flexor pronator mass and through the two heads of the flexor carpi ulnaris. The median nerve was also abnormal, with mild hyperintensity and minimal perineural fibrosis, which gradually normalized as it traversed beneath and distal to the pronator teres fascial edge. Additionally, the medial antebrachial cutaneous nerve (MABC) was focally enlarged in the distal arm, suggesting a neuroma. No regional muscle denervation changes were apparent in the arm or visualized in the proximal forearm.

The patient subsequently underwent repeat ulnar nerve surgery, at which time the MRN findings were confirmed. These included focal fibrosis-related ulnar nerve re-entrapment, median nerve entrapment and MABC neuroma (Figures $2 \mathrm{~A}$ to $2 \mathrm{D}$ ). The ulnar and median nerves were neurolyzed and the MABC neuroma was resected. The pronator muscle and its thickened fascia were partially resected.

${ }^{1}$ Musculoskeletal Section, Russell H Morgan Department of Radiology and Radiology Science; ${ }^{2}$ Departments of Plastic Surgery and Neurosurgery, Johns Hopkins University School of Medicine, Baltimore, Maryland, USA

Correspondence: Dr Avneesh Chhabra, The Russell H Morgan Department of Radiology and Radiological Science, The Johns Hopkins Hospital, 601 North Caroline Street, Baltimore, Maryland 21287, USA. Telephone 443-614-6179, fax 443-287-6438,

e-mail achhabr6@jhmi.edu 

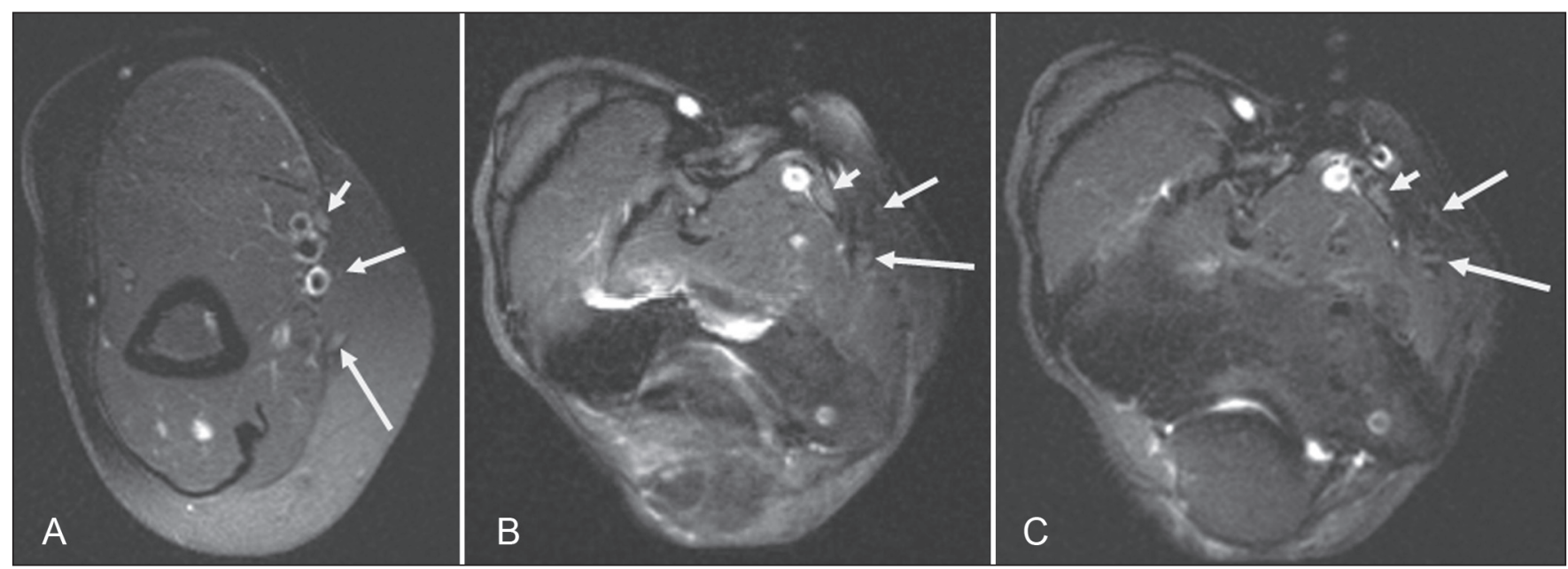

Figure 1) Sequential axial T2 spectral adiabatic inversion recovery images through the distal arm (A) and elbow (B and C) show the hyperintense and mildly enlarged median nerve (short arrows), medial antebrachial cutaneous nerve (medium arrows) and ulnar nerve (long arrows). Notice perineural fibrosis (hypointensity) around the ulnar nerve (A, B and $\mathbf{C})$ and median nerve $(\mathrm{C})$ and mild flattening of the ulnar and median nerves as they traverse posteriorly through the flexor-pronator muscle (C)
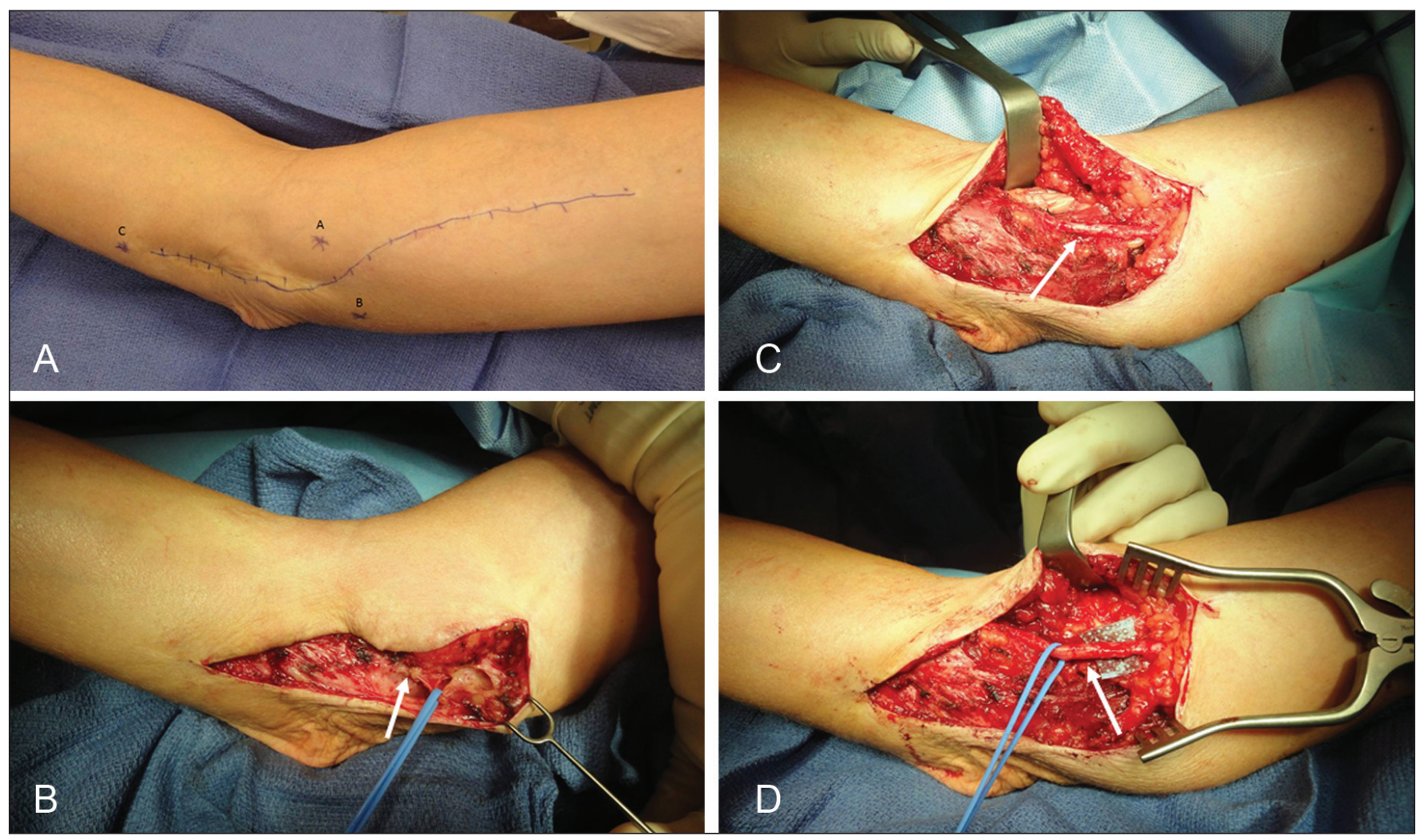

Figure 2) A External photograph with landmarks used before surgery. Notice the soft tissue gap related to scarring from previous injury and surgery between point A (humeral epicondyle) and B (triceps margin). The neuroma of medial antebrachial cutaneous was expected at point B, which was also the pain area. Point $\mathrm{C}$ would be where the nerve will re-enter the muscles. B Intraoperative photograph. Medial antebrachial cutaneous nerve (blue loop) entering the neuroma (arrow). C Intraoperative photograph. Postneurolysis, minimally hyperemic median nerve (arrow) following removal of the overlying scarring. D Intraoperative photograph. Postneurolysis, moderately hyperemic ulnar nerve (arrow) following removal of the overlying scarring. The neurolyzed ulnar nerve is seen proximally and across the elbow joint, from where it traverses posterior through the muscle mass and scarred, re-attached flexor-pronator muscle

\section{Patient 2}

A 52-year-old man with a history of two previous ulnar release surgeries (in situ release and anterior subcutaneous transposition) presented with symptoms of persistent right arm and elbow pain, tingling sensation in the ring and little fingers, and weakness of pinch and grip. The Tinel sign was positive at the anterior elbow. EMG showed moderate ulnar neuropathy. The patient underwent 3T MRN of the right brachial plexus, right arm and elbow to evaluate the status of the ulnar nerve. The MRN demonstrated normal appearance of the brachial plexus and ulnar nerve in the arm. Proximal to the elbow joint, marked hyperintensity and enlargement of the ulnar nerve was noted with enlarged fascicles, which started at the level of the distal humeral metaphysis. There were two focal areas of abrupt transition in the course of the ulnar nerve secondary to the presence of surrounding fascial thickening and scar tissue. The first area of focal narrowing was at the level of the humeral condyles and the second was where the 

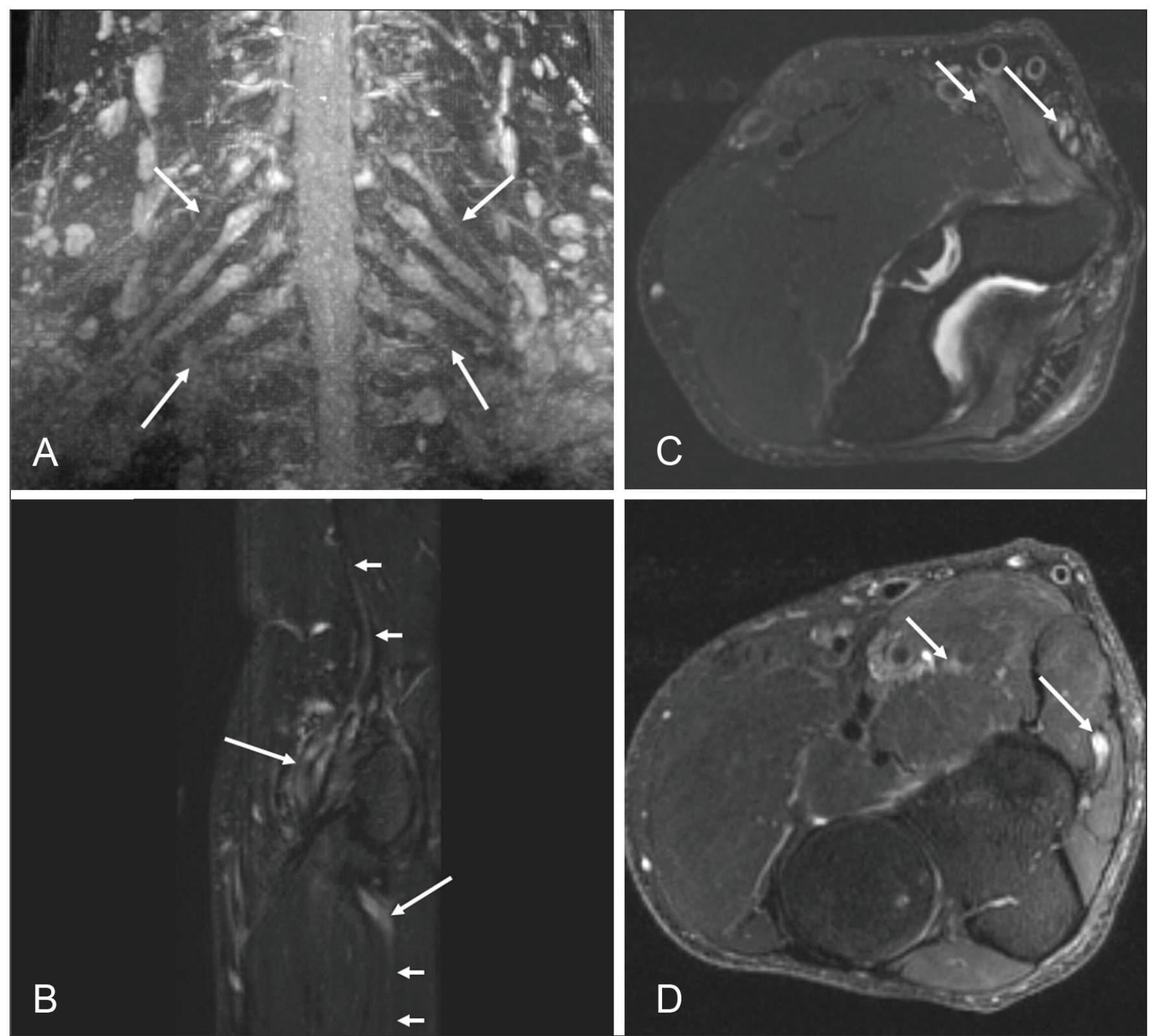

Figure 3) A Coronal three-dimensional maximum intensity projection STIR SPACE (sampling perfection with application optimized contrast using varying flip angle evolutions) image of the brachial plexus shows normal symmetrical appearance of nerves bilaterally. B Oblique sagittal reconstruction from threedimensional SPACE sequence through the elbow shows enlarged ulnar nerve with focal course deviations (long arrows). Notice normal nerve above and below (outlined by short arrows). Sequential axial T2 spectral presaturation attenuated inversion-recovery images through the right elbow (C) and proximal forearm (D) confirm the scar entrapped ulnar nerve with fascicular enlargement (long arrows); also notice mildly abnormal, hyperintense and enlarged median nerve (short arrow in $\mathrm{D}$ ) immediately proximal to the pronator teres fascial edge

nerve traversed from the subcutaneous fat posteriorly into the flexor carpi ulnaris muscle (Figures 3A to 3D). Distally, while coursing through the flexor carpi ulnaris muscle heads, the nerve normalized in size and signal. The median nerve was minimally hyperintense and enlarged immediately proximal to the flexor pronator fascial edge. The median nerve was normal distally. The findings accurately correlated intraoperatively, during which both ulnar and median nerves were found to be entrapped (Figure 4). The enlargement of ulnar nerve, fascicular enlargement, scar entrapment and its abnormal course were confirmed. The median nerve was entrapped in scarring at the fascial edge of the pronator teres. The ulnar and median nerves were neurolyzed, and the pronator muscle and its thickened fascia were partially resected. Both patients did well at their four-week follow-up visit, with significantly reduced pain and sensory symptoms.

\section{DISCUSSION}

The ulnar nerve arises from the medial cord of the brachial plexus with predominant contribution from the C8-T1 nerve roots. The nerve courses through the axilla and descends along the medial border of the medial head of the triceps. The nerve descends posteriorly into the cubital tunnel, a fibro-osseous canal confined between the medial humeral epicondyle, the olecranon process, and a wide band of fibrous tissue known as the cubital tunnel retinaculum. This fibro-osseous canal is the second most common site of ulnar nerve entrapment in the upper extremity after carpal tunnel syndrome. The medial antebrachial cutaneous nerve of the arm also arises from the medial cord. It lies medial to the axillary vein and brachial artery coursing distally in a relatively straight trajectory and supplies the anterior lower one-third of the arm and elbow joint. The median nerve arises from the lateral cord (C5-C7 roots) and medial cord (C8-T1 
roots) of the brachial plexus. All of these nerves follow a relatively straight course through the arm without abrupt angulations (3).

Surgical outcomes after ulnar nerve decompression at the elbow have been reported to be unfavourable compared with carpal tunnel syndrome $(1,2)$. The findings at revision surgery may show dense fibrotic tissue tethering of the ulnar nerve, pseudoneuroma formation, flattening of the nerve, subluxation of the nerve, thickened medial intermuscular septum, and kinking/abnormal angulation of the ulnar nerve $(2,6)$. Occasionally, previous surgery or intervention-related neuroma or entrapment of the medial antebrachial cutaneous nerve may mimic recurrent ulnar neuropathy. The two cases show most of these findings. Entrapment of the median nerve was likely due to previous surgery-related fibrosis in the area and was not suspected preoperatively until MRN revealed the abnormality.

Symptoms indicative of recurrent ulnar nerve entrapment include pain, tingling, numbness, or cold intolerance in the ring and little finger, which may be associated with pain and a positive Tinel sign at the elbow and weakness in the forearm or hand (7). Symptoms can sometimes be nonspecific or nonlocalizing, and the situation may become clinically challenging if multiple previous interventions have been performed. While contemplating repeat surgery, the surgeon needs to know about nerve anatomy, continuity of the nerve, exact site of re-entrapment, status of other regional nerves and other findings. 3T MRN is frequently being used to evaluate such cases due to its ability to show normal and abnormal nerves, and the causative perineural lesion (8). The sensitivity and specificity of MRN for ulnar nerve entrapment on qualitative assessment has been demonstrated to be $83 \%$ and $85 \%$, respectively (9). In these cases, MRN not only localized the nerve abnormality but also detected multiple other nerve abnormalities that accurately correlated with surgical findings. In addition, MRN excluded joint derangement and thoracic outlet syndrome findings, which could have mimicked or exacerbated the symptoms. In both cases, median nerve abnormalities were not apparent on EMG and surgically it was found to be entrapped, similar to MRN findings. EMG is also not very useful in the diagnosis of thoracic outlet syndrome due to the deep location of the nerves. Thus, MRN can be very useful to outline the anatomy, nerve abnormality and depiction of normal nerves in the thoracic outlet, which helps to avoid unnecessary surgery in the latter location. Finally, objective depiction of nerve abnormality on imaging leads to more diagnostic confidence on the part of the surgeon and leads to correct surgical approach, thereby impacting patient morbidity.

To our knowledge, previous injury or surgery-related neuroma of the medial antebrachial cutaneous nerve on MRN has not been previously demonstrated. The medial antebrachial cutaneous nerve is normally difficult to visualize on MRN due to its small size. However, abnormal nerve enlargement, as in the present case, should make it easily apparent. A good understanding of regional nerve anatomy and possible pathologies is essential for prospective observation of such findings.

3T MRN is a very useful examination to evaluate 'failed' or recurrent cases of cubital tunnel syndrome. Multinerve and multifocal

\section{REFERENCES}

1. Kose KC, Bilgin S, Cebesoy O, et al. Clinical results versus subjective improvement with anterior transposition in cubital tunnel syndrome. Advance Thera 2007;24:996-1005.

2. Dellon AL. Techniques for successful management of ulnar nerve entrapment at the elbow. Neurosurg Clin N Am 1991;2:57-73.

3. Mackinnon SE, Dellon AL. Surgery of the Peripheral Nerve. New York: Thieme Medical Publishers, 1988.

4. Chhabra A, Chalian M, Soldatos T, et al. 3-T high-resolution MR neurography of sciatic neuropathy. AJR Am J Roentgenol 2012;198:W357-64.

5. Lee PP, Chalian M, Bizzell C, et al. Magnetic resonance neurography of common peroneal (fibular) neuropathy. J Comp Assist Tomography 2012;36:455-61.

6. Bartels RH, Grotenhuis JA. Anterior submuscular transposition of the ulnar nerve. For post-operative focal neuropathy at the elbow. J Bone Joint Surg 2004;86:998-1001.

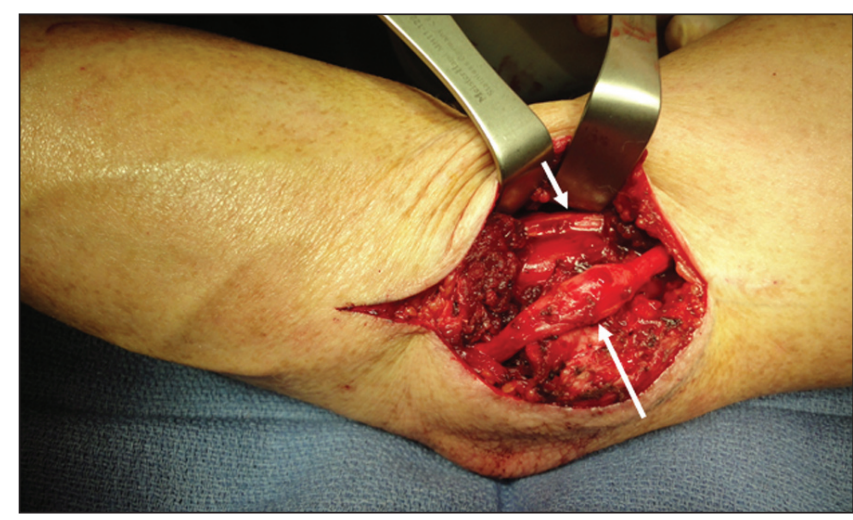

Figure 4) Intraoperative photograph. Hyperemic ulnar nerve (long arrow) and median nerve (short arrow), status post neurolysis. Notice persistent enlargement of the ulnar nerve

abnormalities may be detected on these examinations. If correctly identified, these observations adequately correlate with intraoperative findings, and offer help to the surgeon. In our practice, MRN is routinely used for preoperative planning of failed extremity nerve release surgeries, and also in primary nerve entrapment planning, when the clinical findings or EMG are noncontributory. MRN can be performed at $1.5 \mathrm{~T}$ or 3T. 3T MRN offers higher signal-to-noise ratio and provides superior image quality. However, if the patient has regional metal in situ, to decrease the susceptibility artefacts, $1.5 \mathrm{~T}$ imaging is preferred over 3T. With high-resolution imaging, MRN guides the surgical approach and detects the organic lesion causing nerve flattening or altered course, and also defines the relationship of the lesion to the nerve. Additionally, MRN can differentiate pseudoneuroma from entrapment, which appears as an enlarged nerve with maintained fascicular appearance versus a true neuroma, which is seen as heterogeneous mass-like appearance with partially effaced or disrupted fascicles, thereby impacting patient management and prognosis $(10,11)$. The abnormal kinking of the ulnar nerve and median antebrachial cutaneous nerve neuroma have also been reported as common findings in surgery in such cases; therefore, the investigator should diligently look for such findings on MRN (12). Finally, normal appearance of brachial plexus resulted in avoidance of unnecessary surgery in that region.

To conclude, MRN supplements information obtained from electrodiagnostic examinations and should be used in the diagnostic algorithm of failed peripheral nerve release surgery for appropriate management planning.

DISCLOSURES: Dr Avneesh Chhabra has research grants from GE-AUR (GERRAF), Siemens Medical Solutions and Integra Life Sciences. Dr Chhabra also serves as a research consultant with Siemens CAD group. Dr John A Carrino has research grants from Siemens Medical Solutions.

7. Williams EH, Dellon AL. Anterior submuscular transposition. Hand clinics 2007;23:345-58, vi.

8. Chhabra A, Andreisek G, Soldatos T, et al. MR neurography: Past, present, and future. AJR Am J Roentgenol 2011;197:583-91.

9. Baumer P, Dombert T, Staub F, et al. Ulnar neuropathy at the elbow: MR neurography - nerve T2 signal increase and caliber. Radiology 2011;260:199-206.

10. Chhabra A, Subhawong TK, Williams EH, et al. High-resolution MR neurography: Evaluation before repeat tarsal tunnel surgery. AJR Am J Roentgenol 2011;197:175-83.

11. Thawait SK, Wang K, Subhawong TK, et al. Peripheral nerve surgery: The role of high-resolution MR neurography. AJNR Am J Neuroradiol 2012;33:203-10.

12. Mackinnon SE, Novak CB. Operative findings in reoperation of patients with cubital tunnel syndrome. Hand (N Y) 2007;2:137-43. 